\title{
Cuff Under Pressure for Greater Accuracy
}

Dean S. Picone ${ }^{1}$, Martin G. Schultz ${ }^{1}$, Alun D. Hughes ${ }^{2}$ \& James E. Sharman ${ }^{1}$

\section{Affiliations}

1. Menzies Institute for Medical Research, College of Health and Medicine, University of Tasmania, Private Bag 23, Hobart, 7000, Australia

2. Institute of Cardiovascular Sciences, University College London, London, UK

Correspondence to Dean S. Picone. 


\section{Abstract}

Purpose of Review

To present the evidence that describes what is being measured by upper-arm cuff blood pressure (BP) and the level of accuracy compared with invasive central aortic and brachial BP. Potential causes of inaccuracy and emerging methods are also discussed.

Recent Findings

On average cuff systolic BP systematically underestimates invasive brachial systolic $\mathrm{BP}$, although in a given individual it may substantially under- or over-estimate central aortic systolic BP. Such errors may affect individual health management outcomes and distort population level data on hypertension prevalence and control.

Oscillometric cuff BP is particularly susceptible to inaccuracy in people with high arterial stiffness and with pathophysiological BP waveform shapes. Emerging cuffless BP methods will be susceptible to inaccuracy if oscillometric cuff BP is used for calibration.

Summary

The original purpose of cuff BP was to estimate central aortic BP. Recent evidence has shown substantial inaccuracy of oscillometric cuff BP exists for the measurement of invasive central aortic and brachial BP. Thus, development of more accurate BP methods, through better understanding of oscillometric and BP waveform morphology, is needed to improve health outcomes related to high BP. Keywords: Hypertension, BP, Blood pressure cuff, Brachial blood pressure, Aortic blood pressure, BP management. 


\section{Introduction}

Raised blood pressure (BP) is the leading cause of disability adjusted life years globally [1]. It is the number one risk factor for cardiovascular disease, which itself is responsible for one in three global deaths [2]. Lowering of BP through lifestyle changes (e.g., exercise and/or diet interventions) or antihypertensive treatment leads to lower risk of adverse events related to BP [3]. Management of BP in clinical practice is guided by measurements taken from upper-arm cuff oscillometric BP devices. However, there remain two major issues regarding the function of oscillometric BP devices: (1) confusion as to what is being measured-do the devices seek to measure central (aortic) BP, that which vital organs are exposed to, or peripheral (brachial) BP, the site of the cuff placement, and (2) significant concerns regarding accuracy. These two highly inter-related issues are important and will be the focus of this review. Potential causes of cuff BP inaccuracy and emerging measurement methods will also be discussed.

\section{Cuff Blood Pressure Measurement Methods}

Measurement of BP using an upper-arm cuff was first described by Scipione RivaRocci in 1896 [4]. Following the discovery of the Korotkoff sounds in 1905 [5], mercury auscultation was used widely in clinical practice, and to this day, it is the non-invasive reference standard in validation studies testing the accuracy of new BP devices [6]. However, mercury auscultation has been phased out of clinical practice due to environmental concerns. In the 1970s, automated oscillometric BP devices were developed [7] and have since proven extremely popular. Oscillometric devices are now recommended for use in clinic, and for ambulatory and home BP monitoring $[8,9,10]$.

Oscillometric algorithms that estimate systolic and diastolic BP are designed to emulate the values derived from mercury auscultation. The oscillometric method works by recording oscillations in cuff pressure usually during the deflation phase, although some devices make recordings during the inflation phase [11•]. The measured oscillations are used to generate an oscillometric envelope. Proprietary algorithms are then applied to detect mean arterial pressure, systolic BP and diastolic BP. Mean arterial pressure is conventionally assumed to be the peak amplitude of the oscillometric envelope, but because algorithms are proprietary, the 
precise method of estimation of systolic and diastolic BP is usually undisclosed [12, 13].

\section{What BP Is the Cuff Meant to Measure?}

In most people, systolic BP and pulse pressure are higher in the large, peripheral arteries than the central aorta $[14,15,16,17,18]$. This phenomenon, known as BP amplification, raises questions on whether cuff devices approximate the central aortic $\mathrm{BP}$, as originally intended [4], or peripheral (brachial) BP. The cuff is applied at the upper-arm because this is the closest artery to the central organs where pressure can safely be measured using the sphygmomanometric cuff inflation approach [4]. It has been argued that the purpose of cuff BP devices is to measure central aortic BP because this is the pressure that the vital organs are exposed to and thus is most clinically relevant $[4,19,20]$. Upper-arm cuff systolic BP may be a reasonable proxy for central aortic systolic BP on average [21,22,23,24], which is probably the reason it provides good prognostic information on future cardiovascular events at a population level [3]. Nevertheless, cuff BP is inaccurate in many individuals [25••], and the extent of inaccuracy may be modified by other factors such as age, sex or co-existing disease $[26,27]$. Improving our understanding of what cuff BP devices measure is critical because there are potential differences in central aortic BP versus brachial BP for associations with clinical outcomes [28], as well as efficacy of antihypertensive treatment [29,30], and this has led to the development of cuff BP devices that purport to measure central BP as distinct from brachial BP [31, 32].

\section{Why Does Inaccurate Cuff BP Matter?}

Inaccurate cuff BP measurement can have adverse implications for individuals and distort population level data on hypertension prevalence. Inaccuracy may manifest as either under- or over-estimates of true BP, and each type of error has different potentially serious clinical implications. At the individual patient level, if true BP is underestimated, the chance may be missed to initiate or up-titrate anti-hypertensive therapy or embed lifestyle changes to reduce BP and lower cardiovascular risk. Alternatively, if true BP is overestimated, there is risk of overtreatment or unnecessary up-titration of anti-hypertensives, which come with cost to the patient and health systems, as well as potential side effects that would otherwise be avoidable. These errors may have the most impact on individuals at intermediate risk 
who have BP on the borderline of hypertension [33] because an inaccurate BP measurement of just a few millimetre $\mathrm{Hg}$ could lead to miscategorization of BP and inappropriate clinical decisions [34].

Some individuals may be at greater risk of inaccurate cuff BP, in particular those with a greater level of vascular disease, such as patients with chronic kidney disease. A recent study of 83 patients with chronic kidney disease examined this issue in the setting of coronary angiography [27•]. In the study, cuff systolic BP underestimated central aortic systolic BP to a greater extent with each advancing level of chronic kidney disease (stage $3(+1.8 \pm 9.9 \mathrm{mmHg})$, stage $4(-2.1 \pm 14.8 \mathrm{mmHg}$ ) and stage $5(-6.5 \pm 9.8 \mathrm{mmHg}))$. Moreover, in the total study population, which also included 41 controls, greater central artery stiffness, which was estimated by carotid-femoral pulse wave velocity, was associated with greater cuff systolic BP underestimation of central aortic systolic BP.

Because greater arterial stiffness is also more prevalent with increasing age [35], a recent retrospective, multicentre study examined whether there was an influence of age on the accuracy of cuff BP by comparison to invasively measured central aortic BP [26]. In 1674 individuals aged 40 to 89 years of age, cuff systolic BP significantly overestimated central aortic systolic BP among people aged in their forties, but in people aged in their seventies and eighties, cuff systolic BP significantly underestimated central aortic systolic BP. Further, cuff diastolic BP overestimated invasive diastolic BP in all age ranges, albeit to a significantly greater extent in older age, and thus, overall there was a profound influence on the accuracy of cuff pulse pressure, which underestimated central aortic pulse pressure at all ages.

At the population level, inaccurate BP measurement could distort data on hypertension prevalence and control rates. A recent study assessed the theoretical change in perceived prevalence and control of hypertension (defined as $\geq 140 / 90$ $\mathrm{mmHg}$ ) caused by inaccurate BP among 1540 adults from rural China [36]. Small underestimation of $\mathrm{BP}$, by just $-4 /-2 \mathrm{mmHg}$, reduced the population prevalence of hypertension by $16 \%$, whilst hypertension control dramatically increased (by $36 \%$ ). Similarly, an overestimation of BP by $+4 /+2 \mathrm{mmHg}$ markedly increased population prevalence of hypertension by $24 \%$ and reduced hypertension control by $30 \%$. These data emphasize that inaccurate cuff BP can theoretically distort statistics on 
hypertension prevalence and control which are critical for appropriate population health planning. In epidemiological studies, foundational knowledge on how systolic $\mathrm{BP}$ and pulse pressure increase and diastolic BP decreases from approximately the 5th decade of life are likely underestimates of the true trends across different age ranges [26].

\section{How Accurate Is Cuff BP for Measuring Intra-arterial BP?}

Our recent individual subject level meta-analysis examined the accuracy of cuff BP for measurement of invasive central aortic BP [25]. The analysis was conducted from data among 39 studies and 1823 subjects (mean age 60 years, 62\% male). On average, cuff systolic BP was not significantly different to central aortic systolic BP. However, there was considerable under- and over-estimation at the individual level, with an overall mean absolute error of $8 \mathrm{mmHg}$. Cuff diastolic BP significantly overestimated central aortic diastolic BP $(+5.5 \mathrm{mmHg})$, which therefore caused significant underestimation of central aortic pulse pressure $(-4.8 \mathrm{mmHg})$. Using the JNC7 hypertension guideline categories [37], concordance of cuff BP and central aortic BP was also assessed. In pre-hypertension (120-139/80-89 mmHg) and stage 1 hypertension ranges (140-159/90-99 mmHg) concordance was only 50$57 \%$. This is concerning, because most of the world's population apparently have BP in this range, [38] albeit acknowledging potential accuracy limitations in this population level data itself. The level of agreement was concerning for cuff versus central aortic systolic BP measurements at thresholds $\pm 5 \mathrm{mmHg}$ ( $33 \%$ agreement), $\pm 10 \mathrm{mmHg}$ (60\% agreement) and $\pm 15 \mathrm{mmHg}$ ( $78 \%$ agreement).

In addition to central aortic BP, the accuracy of cuff BP compared with invasive brachial BP was also examined in the meta-analysis. There is a general expectation that brachial cuff BP represents measures of invasive brachial BP, which appears logical because it is the site of cuff inflation. This assumption may be questionable because inflation of the cuff occludes blood flow, thus altering local brachial haemodynamics. Indeed, contrary to belief, invasive brachial systolic BP and diastolic BP were systematically under- and over-estimated, by an average of -5.7 $\mathrm{mmHg}$ and $+5.5 \mathrm{mmHg}$ respectively. Overall, on average, this meant that cuff pulse pressure substantially underestimated the true (intra-arterial) brachial pulse pressure $(-12.0 \mathrm{mmHg})$. Altogether, the results from this meta-analysis show that cuff systolic 
BP (1) is inaccurate in around $50 \%$ of people with systolic BP in the range of prehypertension and stage 1 hypertension (120-160 mmHg), (2) substantially underand over-estimates central aortic systolic BP (random error) and (3) systematically underestimates invasive brachial systolic BP. These data demonstrate the need to understand causes of cuff BP inaccuracy and find appropriate solutions.

\section{Potential Causes of Cuff BP Inaccuracy}

As discussed earlier, there is an influence of age on the accuracy of cuff BP. There is a progressive change in the accuracy of cuff systolic BP, from substantial overestimation of invasive aortic systolic BP in children and adolescents [39], through to greater underestimation with increasing age in older adults [26]. On the other hand, cuff diastolic BP is relatively accurate in children and adolescents, but progressively overestimates invasive diastolic BP with increasing age in adults. These errors in the accuracy of systolic BP and diastolic BP lead to marked inaccuracy of cuff pulse pressure for measurement of invasive aortic pulse pressure in both children and adolescents (cuff overestimates) and older adults (cuff underestimates). It is unclear whether these errors are attributable to pathophysiology (e.g., heightened arterial stiffness/disease in older age), some other heretofore undefined issue (e.g., changes in soft tissue characteristics of the upper arm), inadequacies of the oscillometric measurement method, or combinations of the above.

Pathophysiological changes to arterial structure and function, such as arterial stiffening and flow abnormalities [40,41], are associated with changes to the morphology of arterial BP waveforms and thus could influence the shape of the oscillometric waveform recording [42]. The peak amplitude of the envelope of the oscillometric waveform is regarded as mean arterial pressure. But, envelopes of oscillometric waveforms are often complex and without a clear peak amplitude, unlike classic textbook figures [39, 42]. Oscillometric waveforms that have broader, ill-defined peaks and less smooth features have been shown to produce less accurate estimates of systolic and diastolic BP than waveforms with a clear peak amplitude and smoother curve [39]. In another, albeit small study of 10 people with normal BP (average age 46 years) compared with 10 people with hypertension (average age 57 years), broader oscillometric waveforms were more common in 
people with hypertension [42]. Because hypertension is commonly seen in older people and those with greater arterial stiffness, these data provide a potential explanation for the inaccuracy of oscillometric cuff systolic and diastolic BP that has been reported with increasing age [26], and arterial stiffness. [27, 43]

Recent work has also demonstrated how individual differences in invasive arterial waveform characteristics are related to cuff BP accuracy [14]. In 126 patients undergoing coronary angiography, four distinct BP phenotypes were discovered based on variation in aortic-to-brachial and brachial-to-radial systolic BP amplification (Fig. 1). Invasive aortic systolic BP was significantly higher in two of the phenotypes, indicative of elevated cardiovascular risk, but the oscillometric cuff systolic BP measurements did not detect this difference.

Across the systolic BP amplification phenotypes there were also significant differences in arterial waveform morphology, despite no differences in cuff BP. This finding further illustrates that differences in waveform morphology could influence the accuracy of cuff BP. Other recent work has also identified systolic BP amplification as a possible reason for inaccuracy of devices that estimate central aortic BP via pulse wave analysis [17]. Moreover, common 'form factor' equations used to derive mean arterial pressure (e.g., diastolic BP + (systolic - diastolic BP) $* 0.33$ or diastolic $\mathrm{BP}+($ systolic - diastolic BP $) * 0.4$ ) are also susceptible to inaccuracy for the measurement of invasive aortic mean arterial pressure due to systolic BP amplification [44]. This finding is probably due to the dependence of the form factor equations on systolic BP and changes in waveform shape across different levels of $\mathrm{BP}$ amplification. Altogether, whilst there has been progress on understanding the potential causes of cuff BP inaccuracy, further mechanistic studies to determine the influence of specific arterial waveform features will be crucial for the development of more accurate BP devices (Table 1). 


\begin{tabular}{lcc}
\hline 1. Why are cuff BP measurements inaccurate? & 2. How can cuff BP be improved? & 3. Testing required for new cuff BP methods. \\
\hline Requires research on: & $\begin{array}{c}\text { Individualized oscillometric algorithms } \\
\text { based on the physiological research in }\end{array}$ & $\begin{array}{c}\text { - Validation according to ISO 81060-2:2018 } \\
\text { for pulse wave analysis devices }\end{array}$ \\
- Associations with oscillometric waveforms & step one & - Clinical testing by determining if the new cuff method \\
- Associations with invasive/non-invasively & podern analytic techniques may be & sefinical outcomes above and beyond \\
recorded BP waveforms & & standard cuff BP \\
- BP amplification & & \\
- Inclusion of select populations to understand & & \\
causes of error & & \\
Elderly & & \\
Individuals with chronic kidney disease & & \\
Individuals with high arterial stiffness & & \\
Children & &
\end{tabular}

$B P$ blood pressure, ISO International Organization for Standardization, ARTERY Association for Research into Arterial Structure and Physiology

\section{Development of More Accurate BP Devices}

The fundamental method of oscillometric cuff BP measurement has changed little since these devices first became commercially available in the 1970s. Some recent efforts to improve the accuracy of oscillometric cuff BP have focused on deeper analysis of arterial waveforms [46, 47]. In one study, [47•] a patient-specific physiological model (which used variables representing brachial artery mechanics) was fitted to the oscillometric waveform with the purpose of improving measurement accuracy across a wide spectrum of BPs. This was a worthwhile objective because oscillometric devices are more inaccurate in people with high pulse pressure and high arterial stiffness [27, 43, 48, 49]. The patient-specific model was trained in 57 subjects and tested among 88 different subjects. In people with high pulse pressure, defined as $\geq 50 \mathrm{mmHg}$, the BP estimated from the patient-specific model was more precise than standard oscillometric cuff devices, as determined by comparison to the reference of invasive brachial BP. In the normal pulse pressure group ( $<50 \mathrm{mmHg}$ ), there was no difference between the patient-specific model and standard oscillometric devices. These findings support the hypothesis that individualized approaches to oscillometry may be useful to improve the accuracy of cuff BP across a wide range of BPs.

Another novel method performed pulse wave analysis on standard oscillometric pressure waves with the purpose of generating an accurate central aortic BP [46]. This is a unique approach because most brachial cuff-based devices that perform pulse wave analysis use an additional inflation to record the pressure waveform. In 191 subjects undergoing coronary angiography, the authors found excellent agreement between the estimated central BP and invasive aortic values for both 
systolic BP (+0.7 $\pm 6.0 \mathrm{mmHg})$ and diastolic BP (+3.0 $\pm 5.2 \mathrm{mmHg})$. Overall, the results from this study place the device as one of the most accurate methods available for the estimation of central BP, albeit noting the importance of replicating the results in other studies. Repeated studies of devices that use mean arterial pressure/diastolic BP for waveform calibration is important because it is wellestablished that the accuracy of estimated central BP from this method of calibration is highly device specific. [24, 50,51$]$

Beyond the standard oscillometric cuff approach, cuff-less BP technologies are rapidly emerging. There are many methods under development including conformal patches, eyewear, ear sensors and wrist-wearable style devices. 'Big tech' companies are also investing in this area, and recently, Samsung had a watch-based cuff-less BP measurement method approved by the South Korean Ministry of Food and Drug Safety [52]. A main limitation of most cuff-less methods, including the Samsung device, is that they require calibration to cuff BP. This means that the inaccuracies from cuff BP are translated to the cuff-less devices. Thus, work to develop cuff calibration-free, accurate cuff-less BP monitoring represents an important ongoing research challenge.

Cuff-less BP measurements from some devices have been compared with cuff BP which implies they are intended to be equivalent [53]. But, cuff-less devices that measure arterial/biometric signals at the wrist may be susceptible to inaccuracy due to brachial-to-radial systolic BP amplification [15, 54]. One recent study examined the issue of brachial-to-radial systolic BP amplification using invasive BP data from 180 individuals undergoing coronary angiography. There was substantial interindividual variability of amplification, whereby $43 \%$ of individuals exhibited little brachial-to-radial systolic BP amplification ( $\leq 5 \mathrm{mmHg}$ ), $46 \%$ of individuals had $>5$ $\mathrm{mmHg}$ amplification, out of which $14 \%$ had $>15 \mathrm{mmHg}$ amplification. Because amplification is highly variable between individuals, it may be necessary to predict the level of amplification to allow correction algorithms to be developed.

Nevertheless, the primary goal of cuff-less devices should be the accurate measurement of central aortic BP because the vital organs are exposed to this BP and thus it is most clinically relevant. 
Another critical step in the development of new BP measurement methods is the validation protocol used to determine accuracy. Each new device must undergo rigorous, standardized validation testing using an internationally accepted protocol that is performed independent of manufacturers $[55,56]$. Validation protocols stipulate inclusion of diverse participant characteristics so that different sexes are represented, with a range of BPs and arm sizes so that the findings are broadly generalizable. There are also specific validation protocols for certain populations where accuracy of BP measurement has been proven to be challenging (e.g., atrial fibrillation, pregnancy). The validation process is critical because most BP devices are non-validated and these are less likely to be accurate than those that have undergone rigorous validation testing $[57,58,59]$. This is also highly relevant to emerging cuff-less methods, and there are specific validation criteria currently under development for these devices. [60]

\section{Key Areas for Future Research}

In this review we have presented evidence that oscillometric cuff BP is inaccurate for the measurement of central aortic BP in many people and that BP waveform morphology and arterial stiffness are probably key causes of inaccuracy. Future research should be targeted toward better understanding of the precise mechanisms of inaccuracy. Novel insights may be gained by using modern modelling techniques such as the reservoir-excess pressure paradigm, which is a physiologically plausible approach to pressure waveform separation. Some of the variables derived from the reservoir-excess pressure model are also related to BP amplification [61], which we have shown is related to BP measurement inaccuracy [14, 17, 44]. These studies will need to include people of different ages, levels of vascular disease and a wide range of BPs to ensure generalizability.

Future work on cuff-less BP devices should focus on achieving accurate measurements of central aortic BP without the need for cuff BP calibration. Additional research on arterial physiology and waveform transmission from the aorta through the entire upper limb will also be beneficial to reaching that research objective. Moreover, there needs to be continued education and advocacy on the importance of rigorous validation of new BP devices, whether cuff-based or cuff-less technologies. 


\section{Conclusions}

The purpose of cuff BP is to approximate central aortic BP, which is the BP that the vital organs are exposed to but which may be quite different from upper arm BP. Oscillometric cuff BP is a reasonable proxy for central aortic BP; however, substantial inaccuracy exists in many individuals and appears to be worse in older age and people with greater arterial stiffness. There is a need for continued research on the relationship between cuff inaccuracy and BP waveform morphology. To date, studies that have leveraged oscillometric and BP waveform information have reported improvements to oscillometric cuff BP accuracy. Work in this area should continue with a goal of developing a highly accurate measurement of central aortic BP. This is crucial because inaccurate cuff BP adversely impacts individual health outcomes and population level information on hypertension prevalence and control. 


\section{References}

Papers of particular interest, published recently, have been highlighted as: • Of importance $\cdot \bullet$ Of major importance

1. GBD Risk Factor Collaborators. Global, regional, and national comparative risk assessment of 84 behavioural, environmental and occupational, and metabolic risks or clusters of risks for 195 countries and territories, 19902017: a systematic analysis for the Global Burden of Disease Study 2017. Lancet. 2018;392(10159):1923-94.

2. Roth GA, Johnson C, Abajobir A, Abd-Allah F, Abera SF, Abyu G, et al. Global, regional, and national burden of cardiovascular diseases for 10 causes, 1990 to 2015. J Am Coll Cardiol. 2017;70(1):1-25.

3. Ettehad D, Emdin CA, Kiran A, Anderson SG, Callender T, Emberson J, et al. Blood pressure lowering for prevention of cardiovascular disease and death: a systematic review and meta-analysis. Lancet. 2016;387(10022):957-67.

4. Zanchetti A, Mancia G. The centenary of blood pressure measurement: a tribute to Scipione Riva-Rocci. J Hypertens. 1996;14(1):1-12.

5. Korotkov NS. Concerning the problem of the methods of blood pressure measurement. J Hypertens. 2005;23(1):5.

6. Stergiou GS, Palatini P, Asmar R, loannidis JP, Kollias A, Lacy P, et al. Recommendations and practical guidance for performing and reporting validation studies according to the universal standard for the validation of blood pressure measuring devices by the Association for the Advancement of Medical Instrumentation/European Society of Hypertension/International Organization for Standardization (AAMI/ESH/ISO). J Hypertens. 2019;37(3):459-66.

7. Ramsey M 3rd. Noninvasive automatic determination of mean arterial pressure. Med Biol Eng Comput. 1979;17(1):11-8.

8. Whelton PK, Carey RM, Aronow WS, Casey DE Jr, Collins KJ, Dennison Himmelfarb C, et al. 2017 ACC/AHA/AAPA/ABC/ACPM/AGS/APhA/ASH/ASPC/NMA/PCNA guideline for the prevention, detection, evaluation, and management of high blood pressure in adults: a report of the American College of Cardiology/American 
Heart Association Task Force on Clinical Practice Guidelines. J Am Coll Cardiol. 2018;71(19):e127-248.

9. Williams B, Mancia G, Spiering W, Agabiti Rosei E, Azizi M, Burnier M, et al. 2018 ESC/ESH guidelines for the management of arterial hypertension: the task force for the management of arterial hypertension of the European Society of Cardiology and the European Society of Hypertension: the task force for the management of arterial hypertension of the European Society of Cardiology and the European Society of Hypertension. J Hypertens. 2018;36(10):1953-2041.

10. Unger T, Borghi C, Charchar F, Khan NA, Poulter NR, Prabhakaran D, et al. 2020 International Society of Hypertension Global Hypertension Practice Guidelines. Hypertension. 2020;75(6):1334-57.

11. Lewis PS, British, Irish Hypertension Society's Blood Pressure Measurement Working P. Oscillometric measurement of blood pressure: a simplified explanation. A technical note on behalf of the British and Irish Hypertension Society. J Hum Hypertens. 2019;33(5):349-51. A useful introductory explanation of the oscillometric method.

12. • Chandrasekhar A, Yavarimanesh M, Hahn JO, Sung $\mathrm{SH}$, Chen $\mathrm{CH}$, Cheng $\mathrm{HM}$, et al. Formulas to explain popular Oscillometric blood pressure estimation algorithms. Front Physiol. 2019;10:1415. Detailed work describing formulas for different oscillometric algorithms.

13. Alpert BS. Can 'FDA-cleared' blood pressure devices be trusted? A call to action. Blood Press Monit. 2017;22(4):179-81.

14. • Picone DS, Schultz MG, Peng X, Black JA, Dwyer N, Roberts-Thomson P, et al. Discovery of new blood pressure phenotypes and relation to accuracy of cuff devices used in daily clinical practice. Hypertension. 2018;71(6):1239-47. A series of blood pressure amplification phenotypes were discovered that have direct relevance to the accuracy of cuff BP. The study highlights the potential importance of using arterial waveform features to improve BP measurement accuracy.

15. Armstrong MK, Schultz MG, Picone DS, Black JA, Dwyer N, RobertsThomson $\mathrm{P}$, et al. Brachial and radial systolic blood pressure are not the same. Hypertension. 2019;73(5):1036-41. 
16. Pauca AL, Wallenhaupt SL, Kon ND, Tucker WY. Does radial artery pressure accurately reflect aortic pressure? Chest. 1992;102(4):1193-8.

17. Picone DS, Schultz MG, Peng X, Black JA, Dwyer N, Roberts-Thomson P, et al. Intra-arterial analysis of the best calibration methods to estimate aortic blood pressure. J Hypertens. 2019;37(2):307-15.

18. Kroeker EJ, Wood EH. Comparison of simultaneously recorded central and peripheral arterial pressure pulses during rest, exercise and tilted position in man. Circ Res. 1955;3(6):623-32.

19. Jankowski P, Kawecka-Jaszcz K, Czarnecka D, Brzozowska-Kiszka M, Styczkiewicz K, Loster M, et al. Pulsatile but not steady component of blood pressure predicts cardiovascular events in coronary patients. Hypertension. 2008;51(4):848-55.

20. Chirinos JA, Zambrano JP, Chakko S, Veerani A, Schob A, Perez G, et al. Relation between ascending aortic pressures and outcomes in patients with angiographically demonstrated coronary artery disease. Am J Cardiol. 2005;96(5):645-8.

21. Narayan O, Casan J, Szarski M, Dart AM, Meredith IT, Cameron JD. Estimation of central aortic blood pressure: a systematic meta-analysis of available techniques. J Hypertens. 2014;32(9):1727-40.

22. Cloud GC, Rajkumar C, Kooner J, Cooke J, Bulpitt CJ. Estimation of central aortic pressure by SphygmoCor requires intra-arterial peripheral pressures. Clin Sci (Lond). 2003;105(2):219-25.

23. Davies JI, Band MM, Pringle S, Ogston S, Struthers AD. Peripheral blood pressure measurement is as good as applanation tonometry at predicting ascending aortic blood pressure. J Hypertens. 2003;21(3):571-6.

24. Schultz MG, Picone DS, Armstrong MK, Black JA, Dwyer N, RobertsThomson $\mathrm{P}$, et al. Validation study to determine the accuracy of central blood pressure measurement using the Sphygmocor Xcel cuff device. Hypertension. 2020;76(1):244-50.

25. •• Picone DS, Schultz MG, Otahal P, Aakhus S, Al-Jumaily AM, Black JA, et al. Accuracy of cuff-measured blood pressure: systematic reviews and metaanalyses. J Am Coll Cardiol. 2017;70(5):572-86 This paper reports on a comprehensive series of individual subject data meta-analyses that describe the accuracy of cuff BP compared with invasive aortic and brachial BP. 
26. Picone DS, Schultz MG, Otahal P, Black JA, Bos WJ, Chen CH, et al. Influence of age on upper arm cuff blood pressure measurement. Hypertension. 2020;75(3):844-50.

27. Carlsen RK, Peters CD, Khatir DS, Laugesen E, Botker HE, Winther S, et al. Estimated aortic blood pressure based on radial artery tonometry underestimates directly measured aortic blood pressure in patients with advancing chronic kidney disease staging and increasing arterial stiffness. Kidney Int. 2016;90(4):869-77. This study found that advanced vascular aging, assessed by carotid-to-femoral pulse wave velocity is associated with worse accuracy of cuff BP.

28. Vlachopoulos C, Aznaouridis K, O'Rourke MF, Safar ME, Baou K, Stefanadis C. Prediction of cardiovascular events and all-cause mortality with central haemodynamics: a systematic review and meta-analysis. Eur Heart J. 2010;31(15):1865-71.

29. Williams B, Lacy PS, Thom SM, Cruickshank K, Stanton A, Collier D, et al. Differential impact of blood pressure-lowering drugs on central aortic pressure and clinical outcomes: principal results of the Conduit Artery Function Evaluation (CAFE) study. Circulation. 2006;113(9):1213-25.

30. Kelly RP, Gibbs HH, O'Rourke MF, Daley JE, Mang K, Morgan JJ, et al. Nitroglycerin has more favorable effects on left-ventricular afterload than apparent from measurement of pressure in a peripheral artery. Eur Heart J. 1990;11(2):138-44.

31. Sharman JE, Laurent S. Central blood pressure in the management of hypertension: soon reaching the goal? J Hum Hypertens. 2013;27(7):405-11.

32. Millasseau S, Agnoletti D. Non-invasive estimation of aortic blood pressures: a close look at current devices and methods. Curr Pharm Des. 2015;21(6):709-18.

33. Sharman JE, Marwick TH. Accuracy of blood pressure monitoring devices: a critical need for improvement that could resolve discrepancy in hypertension guidelines. J Hum Hypertens. 2019;33(2):89-93.

34. Jones DW, Appel LJ, Sheps SG, Roccella EJ, Lenfant C. Measuring blood pressure accurately: new and persistent challenges. JAMA. 2003;289(8):1027-30. 
35. Reference Values for Arterial Stiffness Collaboration. Determinants of pulse wave velocity in healthy people and in the presence of cardiovascular risk factors: 'establishing normal and reference values'. Eur Heart J. 2010;31(19):2338-50.

36. Campbell NRC, Padwal R, Picone DS, Su H, Sharman JE. The impact of small to moderate inaccuracies in assessing blood pressure on hypertension prevalence and control rates. J Clin Hypertens (Greenwich). 2020;22(6):939_ 42.

37. Chobanian AV, Bakris GL, Black HR, Cushman WC, Green LA, Izzo JL Jr, et al. The Seventh Report of the Joint National Committee on Prevention, Detection, Evaluation, and Treatment of High Blood Pressure: the JNC 7 report. JAMA. 2003;289(19):2560-72.

38. Danaei G, Finucane MM, Lin JK, Singh GM, Paciorek CJ, Cowan MJ, et al. National, regional, and global trends in systolic blood pressure since 1980: systematic analysis of health examination surveys and epidemiological studies with 786 country-years and 5.4 million participants. Lancet. 2011;377(9765):568-77.

39. Amoore JN, Lemesre Y, Murray IC, Mieke S, King ST, Smith FE, et al. Automatic blood pressure measurement: the oscillometric waveform shape is a potential contributor to differences between oscillometric and auscultatory pressure measurements. J Hypertens. 2008;26(1):35-43.

40. Hashimoto J, Westerhof BE, Ito S. Carotid flow augmentation, arterial aging, and cerebral white matter hyperintensities. Arterioscler Thromb Vasc Biol. 2018;38(12):2843-53.

41. Hashimoto J, Ito S. Pulse pressure amplification, arterial stiffness, and peripheral wave reflection determine pulsatile flow waveform of the femoral artery. Hypertension. 2010;56(5):926-33.

42. Zheng DC, Giovannini R, Murray A. Asymmetrical oscillometric pulse waveform envelopes in normotensive and hypertensive subjects. Computing in Cardiology. 2010;37:377-80.

43. Kuwajima I, Hoh E, Suzuki Y, Matsushita S, Kuramoto K. Pseudohypertension in the elderly. J Hypertens. 1990;8(5):429-32. 
44. Schultz MG, Picone DS, Armstrong MK, Black JA, Dwyer N, RobertsThomson P, et al. The influence of SBP amplification on the accuracy of formfactor-derived mean arterial pressure. J Hypertens. 2020;38(6):1033-9.

45. Sharman JE, Avolio AP, Baulmann J, Benetos A, Blacher J, Blizzard L, et al. Validation of non-invasive central blood pressure devices: artery society task force consensus statement on protocol standardization. Eur Heart J. 2017;38(37):2805-12.

46. • Dorr M, Richter S, Eckert S, Ohlow MA, Hammer F, Hummel A, et al. Invasive validation of antares, a new algorithm to calculate central blood pressure from oscillometric upper arm pulse waves. J Clin Med. 2019;8(7). This paper is a validation study according to the ARTERY statement. The novel device that was tested performs pulse wave analysis on oscillometric BP waveforms instead of waveforms recorded at a static BP level.

47. • Liu J, Cheng HM, Chen CH, Sung SH, Hahn JO, Mukkamala R. Patientspecific Oscillometric blood pressure measurement: validation for accuracy and repeatability. IEEE J TransI Eng Health Med. 2017;5:1900110. Proof-ofprinciple study that developed a more individualised approach to oscillometric BP measurement which improved precision in people with high pulse pressure.

48. van Popele NM, Bos WJ, de Beer NA, van Der Kuip DA, Hofman A, Grobbee $\mathrm{DE}$, et al. Arterial stiffness as underlying mechanism of disagreement between an oscillometric blood pressure monitor and a sphygmomanometer. Hypertension. 2000;36(4):484-8.

49. Stergiou GS, Lourida P, Tzamouranis D, Baibas NM. Unreliable oscillometric blood pressure measurement: prevalence, repeatability and characteristics of the phenomenon. J Hum Hypertens. 2009;23(12):794-800.

50. Weber T, Wassertheurer S, Rammer M, Maurer E, Hametner B, Mayer CC, et al. Validation of a brachial cuff-based method for estimating central systolic blood pressure. Hypertension. 2011;58(5):825-32.

51. Pucci G, Vaudo G, Picone DS. Accuracy of non-invasive central BP estimation: still a long "wave" to go. J Hypertens. 2020;in press.

52. Samsung. Samsung announces blood pressure monitoring application for Galaxy watch devices: Samsung; 2020 [updated 21 April 2020. Available 
from: https://news.samsung.com/global/samsung-announces-blood-pressuremonitoring-application-for-galaxy-watch-devices.

53. Bilo G, Zorzi C, Ochoa Munera JE, Torlasco C, Giuli V, Parati G. Validation of the Somnotouch-NIBP noninvasive continuous blood pressure monitor according to the European Society of Hypertension International Protocol revision 2010. Blood Press Monit. 2015;20(5):291-4.

54. Armstrong MK, Schultz MG, Picone DS, Black JA, Dwyer N, RobertsThomson P, et al. Response by Armstrong et al to letter regarding article "brachial and radial systolic blood pressure are not the same: evidence to support the Popeye phenomenon". Hypertension. 2019;74(2):e35-e6.

55. BS EN ISO 81060-3. Non-invasive sphygmomanometers. Part 3. Clinical investigation of continuous non-invasive automated measurement type. [Available from: https://shop.bsigroup.com/ProductDetail?pid=000000000030397676. Accessed 11 July 2019.

56. Sharman JE, O'Brien E, Alpert B, Schutte AE, Delles C, Hecht Olsen M, et al. Lancet commission on hypertension group position statement on the global improvement of accuracy standards for devices that measure blood pressure. J Hypertens. 2020;38(1):21-9.

57. Jung MH, Kim GH, Kim JH, Moon KW, Yoo KD, Rho TH, et al. Reliability of home blood pressure monitoring: in the context of validation and accuracy. Blood Press Monit. 2015;20(4):215-20.

58. Akpolat T, Dilek M, Aydogdu T, Adibelli Z, Erdem DG, Erdem E. Home sphygmomanometers: validation versus accuracy. Blood Press Monit. 2009;14(1):26-31.

59. Picone DS, Deshpande RA, Schultz MG, Fonseca R, Campbell NRC, Delles $\mathrm{C}$, et al. Nonvalidated home blood pressure devices dominate the online marketplace in Australia: major implications for cardiovascular Risk management. Hypertension. 2020;75(6):1593-9.

60. Sharman JE, O'Brien E, Alpert B, Delles C, Hecht Olsen M, Mcmanus RJ, et al. Reply. J Hypertens. 2020;38(4):775.

61. Peng X, Schultz MG, Picone DS, Black JA, Dwyer N, Roberts-Thomson P, et al. Arterial reservoir characteristics and central-to-peripheral blood pressure amplification in the human upper limb. J Hypertens. 2017;35(9):1825-31. 


\section{Conflict of Interest}

Dr. Sharman reports his university has received equipment and research funding from manufacturers of blood pressure devices including AtCor Medical, IEM and Pulsecor (Uscom). He has no personal, financial or commercial interests related to blood pressure device companies. The other authors declare no conflicts of interest relevant to this manuscript.

\section{Human and Animal Rights and Informed Consent}

This article does not contain any studies with human or animal subjects performed by any of the authors. 

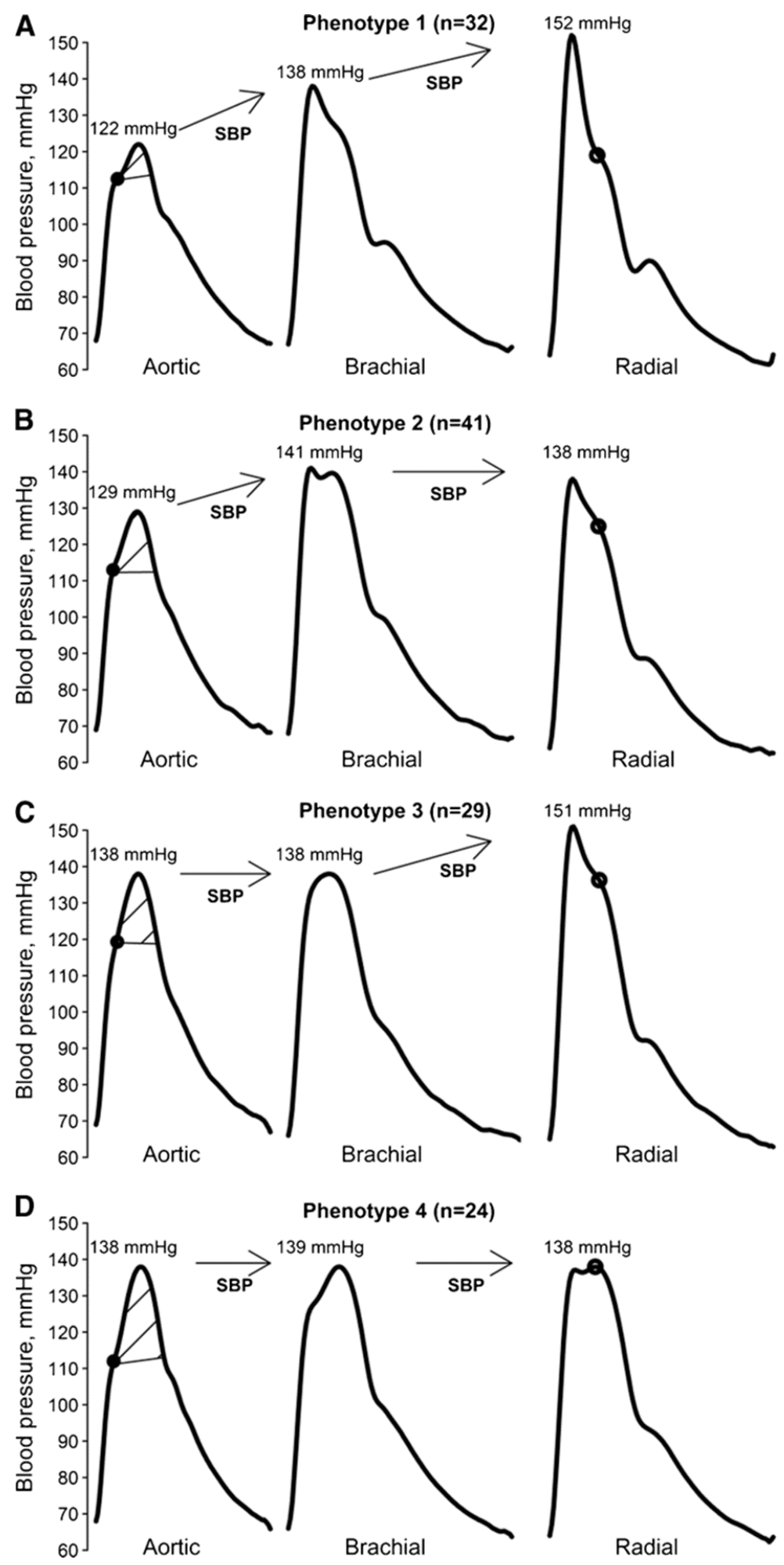

Fig. 1 Systolic BP amplification phenotypes discovered from variability between the aorta-to-brachial-to-radial arteries [14]. Crucially, cuff BP was not different between the four phenotypes despite significant differences in aortic BP. Republished according to the AHA Copyright Transfer Agreement 